preventing to lie down for many hours were excluded. In the operating, the patients received a bolus dose of dexmedetomidine $1 \mu \mathrm{g} / \mathrm{kg}$ over 10 minutes followed by an infusion of $0.2-0.7 \mu \mathrm{g} / \mathrm{kg} / \mathrm{hr}$ (BIS target 60-80). Once the patients were sedated, scalp block was given with bupivacaine $0.25 \%$. The data on hemodynamics at various painful stages of the procedure, intraoperative complications, nausea/vomiting, brain bulge, seizures etc., were collected. Total amount of fentanyl used, intravenous (IV) fluids required, blood loss and transfusion, duration of surgery, ICU and hospital stay were also recorded. Postoperatively, the patient's satisfaction was assessed using numerical rating scale. Results: A total of 13 patients underwent awake craniotomy during a period of one year. The mean age and weight was 38.7 years and $66.1 \mathrm{~kg}$, respectively. The intraoperative vitals remained stable in all patients. None of the patients experienced any episode of desaturation. One patient developed brain bulge and was managed with propofol. The average fentanyl consumption was $200 \mu \mathrm{g}$. The duration of surgery, ICU and hospital stays were 6 hours, 1 and 5 days, respectively. The overall patient satisfaction score was 8 . Conclusion: The use of dexmedetomidine infusion with scalp block for awake craniotomies is a safe and efficacious. Absence of complications and high patient satisfaction score makes it close to an ideal agent for awake craniotomy.

\section{Randomized controlled single blinded prospective study to evaluate the effect of bispectral index (BIS) guided sevoflurane on emergence in neurosurgical patients undergoing craniotomy over the conventional method}

\section{R. Rajani, Sumit kalra, Joseph N. Monteiro, Ratan Chelani, Borges, Manju Butani \\ Department of Anesthesiology, P.D. Hinduja National Hospital and Medical Research Centre, Mumbai, Maharashtra, India}

Background: Smooth extubation, haemodynamic stability, rapid emergence from anaesthesia allow immediate neurological evaluation. We evaluated the efficacy of BIS guided use of sevoflurane till the head dressing on emergence in neurosurgical patients undergoing craniotomy over the conventional method. Materials and Methods: Sixty ASA I and II patients, aged 18-65 yrs, undergoing craniotomy under anaesthesia were randomly divided into two groups using computer generated table. The standard neuroanesthesia protocols and drugs were used for monitoring, induction and maintenance of anesthesia. Group C-patients given sevoflurane till the end of last skin suture. Group S-patients given sevoflurane till the end of head dressing keeping BIS between 40-60. After surgery extubation was done with reversal of neuromuscular blockade. In both groups heart rate, mean arterial pressure were recorded until the extubation and10 minutes post extubation. Patients' response to awakening time and response to verbal commands and aldrette score were also noted. Results: All data measured and analyzed using standard statistical tests (student's $t$-test, Chi-square, Fisher's Exact Probability tests, Man- Whitney, Wilcoxon rank test). A ' $P$ ' value of $\leq 0.05$ was taken as significant. The mean arterial-pressure and heart rate were comparable in both groups. The need for rescue medications (nitroglycerine for hypertension, esmolol for tachycardia) was higher in $C$ group. Extubation time and emergence time were comparable in both groups. Conclusion: There is no significant difference in hemodynamic response and emergence time in the BIS guided sevoflurane use till the head dressing over the conventional method.

\section{Single low dose dexmedetomidine for attenuation of hemodynamic responses and airway reflexes during extubation following spine surgery - A randomized controlled prospective study}

\author{
Sumit Kalra, M. R. Rajani, Joseph N. Monteiro, \\ Pramila Kurkal, Manju Butani \\ Department of Anesthesiology, P.D. Hinduja National \\ Hospital and Medical Research Centre, Mumbai, \\ Maharashtra, India
}

Background:Smooth extubation, haemodynamic stability, rapid emergence from anaesthesia allow immediate neurological evaluation. We evaluated the efficacy of single low dose dexmedetomidine $(0.25 \mathrm{mcg} / \mathrm{kg})$ vs. normal saline for attenuation of hemodynamic responses and airway reflexes during extubation following spine surgery. Materials and Methods: Eighty ASA I and II patients, aged 18-65 years, undergoing spine surgery under anesthesia were randomly divided into two groups using computer generated table. The standard neuroanesthesia protocols and drugs were used for monitoring, induction and maintenance of anesthesia. After discontinuing sevoflurane, 5 minutes before end of surgery, patients in Group D received dexmedetomidine $0.25 \mathrm{mcg} / \mathrm{kg}$ and Group C received $20 \mathrm{ml}$ normal saline intravenously over 5 minutes. With patient supine, extubation was done after reversal of neuromuscular blockade. Heart rate, oxygen saturation, Systolic and Diastolic Blood Pressure were recorded before administration of the drug and at 1 , 3 and 5 minutes during drug administration, at completion of dressing, at the time of extubation, and at 1, 3, 5, 7 and 